\title{
Homogeneity and Markovity of electronic dephasing in liquid solutions
}

\author{
Being J. Ka, Ming-Liang Zhang, and Eitan Geva ${ }^{a)}$ \\ Department of Chemistry, University of Michigan, 930 North University, Ann Arbor, \\ Michigan 48109-1055
}

(Received 13 June 2006; accepted 21 August 2006; published online 29 September 2006)

\begin{abstract}
The electronic dephasing dynamics of a solvated chromophore is formulated in terms of a non-Markovian master equation. Within this formulation, one describes the effect of the nuclear degrees of freedom on the electronic degrees of freedom in terms of a memory kernel function, which is explicitly dependent on the initial solvent configuration. In the case of homogeneous dynamics, this memory kernel becomes independent of the initial configuration. The Markovity of the dephasing process is also the most conveniently explored by comparing the results obtained via the non-Markovian master equation to these obtained via its Markovian counterpart. The homogeneous memory kernel is calculated for a two-state chromophore in liquid solution, and used to explore the sensitivity of photon echo signals to the heterogeneity and non-Markovity of the underlying solvation dynamics. (C) 2006 American Institute of Physics. [DOI: 10.1063/1.2354155]
\end{abstract}

\section{INTRODUCTION}

The electronic energy levels of a dilute chromophore in solution are very sensitive to the configuration of solvent atoms in its vicinity. Solvent dynamics therefore leads to fluctuations in the chromophore transition frequency, which is known as spectral diffusion. As is well known, spectral diffusion can lead to the relaxation of electronic coherences which is known as dephasing or decoherence. By studying the rate of dephasing, one can therefore learn about the dynamics of the solvent in the vicinity of the chromophore.

The time scale on which spectral diffusion occurs is often related to the inverse of a homogeneous linewidth. The latter is clearly distinguished from the inhomogeneous linewidth brought about by the equilibrium distribution of solvent configurations. In many systems of practical interest, the absorption line shape is dominated by inhomogeneous broadening, and is therefore insensitive to spectral diffusion. The latter can be studied with the help of various nonlinear spectroscopic techniques, such as photon echoes. ${ }^{1-6}$ However, these techniques still involve ensemble averaging over a very large number of chromophore molecules. This averaging makes it difficult to distinguish between the case of heterogeneous dynamics, which would be strongly dependent on the initial configuration, and the case of homogeneous dynamics, which would not.

To what extent is the dephasing experienced by different chromophore molecules throughout the sample truly homogeneous? This question is easily answered in cases where the bath can be represented by delocalized excitations, such as acoustic phonons. The dynamics in such cases is truly homogeneous since different chromophore molecules throughout the host matrix are coupled to the very same phonon bath. ${ }^{7}$ Dynamical heterogeneity of relatively slow spectral diffusion processes, in such systems as disordered crystals and glasses, can also be probed directly by single-molecule spectroscopic

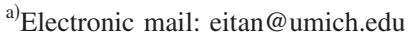

techniques. ${ }^{8-14}$ However, the situation is different in the case of liquid solutions, where electronic dephasing is usually ultrafast and may be highly correlated with the initial configuration. The fact that the dephasing dynamics occurs on the same time scale as that of the nuclear degrees of freedom (DOF) also suggests that it is non-Markovian.

In this paper, we propose a general theoretical framework for quantitatively studying the homogeneity and Markovity of dephasing processes. Whereas previous studies have considered non-Markovian effects in dephasing dynamics within the framework of phenomenological stochastic models, ${ }^{15-19}$ the scheme proposed here is more general and based on describing the dephasing process in terms of a nonMarkovian generalized quantum master equation. A central role within this formulation is played by the memory kernel, which is explicitly dependent on the initial solvent configuration. In the case of homogeneous dynamics, this memory kernel becomes independent of the initial configuration. One may then explore the degree to which the dephasing process is homogeneous by comparing the results computed by assuming a homogeneous memory kernel to these computed without this assumption. The Markovity of the dephasing process is also most conveniently explored by comparing results computed via the non-Markovian master equation to these computed via its Markovian counterpart.

The remainder of this paper is organized as follows. The formalism required for describing dephasing processes is presented in Sec. II. The general non-Markovian master equation underlying dephasing is derived in Sec. III. The formalism required for calculating nonlinear photon echo signals is described in Sec. IV. The application of the theoretical framework for studying Markovity and homogeneity of dephasing in nonpolar liquid solutions is reported in Sec. V. We conclude with a summary of the main results in Sec. VI. 


\section{PRELIMINARY CONSIDERATIONS}

We consider a system with an overall Hamiltonian of the following generic form (capped symbols, such as $\hat{A}$, represent operators throughout this paper):

$$
\hat{H}=\hat{H}_{g} \otimes|g\rangle\left\langle g\left|+\left(\hbar \omega_{\mathrm{eg}}+\hat{H}_{e}\right) \otimes\right| e\right\rangle\langle e| .
$$

Here, $|g\rangle$ and $|e\rangle$ represent the ground and excited electronic states, respectively, $\hat{H}_{g}$ and $\hat{H}_{e}$ represent the corresponding quantum-mechanical Hamiltonians of the nuclear DOF, and $\hbar \omega_{\text {eg }}$ is the energy gap between the two bare electronic states. Off-diagonal coupling terms between the electronic states were left out in light of the fact that electronic population relaxation rates in liquid solutions are typically considerably slower in comparison to the ultrafast electronic dephasing rates. We also assume that the initial state is given by

$$
\begin{aligned}
\hat{\rho}(0)= & \hat{\rho}_{b} \otimes\left(\sigma_{g g}(0) \otimes|g\rangle\left\langle g\left|+\sigma_{e e}(0) \otimes\right| e\right\rangle\langle e|\right. \\
& \left.+\sigma_{g e}(0) \otimes|g\rangle\left\langle e\left|+\sigma_{\mathrm{eg}}(0) \otimes\right| e\right\rangle\langle g|\right) .
\end{aligned}
$$

Here, $\hat{\rho}_{b}$ is a density operator representing the initial state of the nuclear DOF. It should be emphasized that $\hat{\rho}_{b}$ does not necessarily coincide with the equilibrium density operator at the ground or excited electronic states, which are given by

$$
\hat{\rho}_{j}^{\mathrm{eq}}=\frac{e^{-\beta \hat{H}_{j}}}{\operatorname{Tr}\left(e^{-\beta \hat{H}_{j}}\right)}, \quad j=g, e .
$$

In many cases of practical interest, one can only directly probe and/or manipulate the electronic DOF. Optical spectroscopy represents a prime example of this state of affairs, where measurable frequency domain spectra and timedomain signals can all be given in terms of expectation values of the purely electronic coherence operators $|g\rangle\langle e|$ and $|e\rangle\langle g|$ (within the Condon approximation). ${ }^{1}$ This observation naturally leads to a strategy that treats the electronic DOF as "the system," and the nuclear DOF as "the bath." To this end, we rewrite the Hamiltonian in Eq. (1) in a system-bath form, where the system and bath correspond to the electronic and nuclear DOFs, respectively,

$$
\hat{H}=\hat{H}_{s}+\hat{H}_{b}+\hat{H}_{b s} .
$$

Here,

$$
\begin{aligned}
& \hat{H}_{s}=\left(\hbar \omega_{\mathrm{eg}}+\langle\hat{U}\rangle_{b}\right)|e\rangle\langle e|, \\
& \hat{H}_{b}=\hat{H}_{g}, \\
& \hat{H}_{b s}=\delta \hat{U} \otimes|e\rangle\langle e|,
\end{aligned}
$$

where $\hat{U}=\hat{H}_{e}-\hat{H}_{g},\langle\hat{U}\rangle_{b}=\operatorname{Tr}_{b}\left(\hat{\rho}_{b} \hat{U}\right)$, and $\delta \hat{U}=\hat{U}-\langle\hat{U}\rangle_{b}$. It should be noted that the system-bath coupling term was conveniently chosen such that $\left\langle\hat{H}_{b s}\right\rangle_{b}=0$, which is also why we defined the system Hamiltonian as $\hat{H}_{s}=\left(\hbar \omega_{\mathrm{eg}}+\langle\hat{U}\rangle_{b}\right)|e\rangle\langle e|$, rather than $\hat{H}_{s}=\hbar \omega_{\mathrm{eg}}|e\rangle\langle e|$.
The state of the overall system at time $t$ is described by the density operator $\hat{\rho}(t)$. The dynamics of $\hat{\rho}(t)$ is dictated by the Liouville equation,

$$
\frac{d}{d t} \hat{\rho}(t)=-\frac{i}{\hbar}[\hat{H}, \hat{\rho}(t)]
$$

The state of the electronic DOF (the system) at time $t$ can be described by the reduced density operator,

$$
\hat{\sigma}(t)=\operatorname{Tr}_{b}[\hat{\rho}(t)] .
$$

For the model under consideration, one can represent $\hat{\sigma}(t)$ by a $2 \times 2$ matrix whose elements are $\sigma_{k l}=\langle k|\hat{\sigma}(t)| l\rangle$, with $k, l$ $=g, e$. It is immediately clear that $\sigma_{g g}(t)=\sigma_{g g}(0)$ and $\sigma_{e e}(t)$ $=\sigma_{e e}(0)$, since $[\hat{H},|g\rangle\langle g|]=[\hat{H},|e\rangle\langle e|]=0$. It can also be shown that

$$
\begin{aligned}
\sigma_{g e}(t) & =\left\langle g\left|\operatorname{Tr}_{b}\left\{e^{-i \hat{H} t / \hbar} \hat{\rho}_{b} \otimes \hat{\sigma}(0) e^{i \hat{H} t / \hbar}\right\}\right| e\right\rangle \\
& =\sigma_{g e}(0) \exp \left\{i\left(\omega_{\mathrm{eg}}+\langle\hat{U}\rangle_{b} / \hbar\right) t\right\} \times J_{b}(t),
\end{aligned}
$$

where

$$
\begin{aligned}
J_{b}(t) & =\left\langle e^{i\left(\hat{H}_{b}+\delta \hat{U}\right) t / \hbar} e^{-i \hat{H}_{b^{t / \hbar}}}\right\rangle_{b} \\
& \equiv\left\langle\exp _{-}\left\{\frac{i}{\hbar} \int_{0}^{t} d t^{\prime} \delta \hat{U}\left(t^{\prime}\right)\right\}\right\rangle_{b} .
\end{aligned}
$$

Here, $\delta \hat{U}(t)=e^{i \hat{H}_{b} t / \hbar} \delta \hat{U} e^{-i \hat{H}_{b} t / \hbar}$ and $\exp _{-}\{\cdots\}$ stands for a negatively time-ordered exponential operator defined by

$$
\begin{aligned}
\exp _{-}\left\{\int_{t_{0}}^{t} d t^{\prime} \hat{A}\left(t^{\prime}\right)\right\} \equiv & 1+\int_{t_{0}}^{t} d t_{1} \hat{A}\left(t_{1}\right) \\
& +\int_{t_{0}}^{t} d t_{1} \int_{t_{0}}^{t_{1}} d t_{2} \hat{A}\left(t_{2}\right) \hat{A}\left(t_{1}\right)+\cdots
\end{aligned}
$$

In practice, it is more convenient to work in terms of the interaction picture. The transformation between $\sigma_{g e}(t)$ and its interaction picture counterpart, $\widetilde{\sigma}_{g e}(t)$, is given by

$$
\widetilde{\sigma}_{g e}(t)=\exp \left\{-i\left(\omega_{\mathrm{eg}}+\langle\hat{U}\rangle_{b} / \hbar\right) t\right\} \sigma_{g e}(t) .
$$

Substituting Eq. (13) into Eq. (10) and rearranging then yields

$$
\frac{\widetilde{\sigma}_{g e}(t)}{\widetilde{\sigma}_{g e}(0)}=J_{b}(t) .
$$

The calculation of the quantum-mechanically exact $J_{b}(t)$ is only feasible in the case of simplified and phenomenological models, such as the Brownian oscillator model. ${ }^{1}$ As a result, a similar calculation for realistic many-body anharmonic systems, such as liquid solutions, usually relies on approximations. In this paper we will employ a simple classical approximation which is often invoked in this 
context. $^{20-23}$ To this end, we approximate the right-hand side of Eq. (11) by its classical limit,

$$
J_{b}^{\mathrm{cl}}(t)=\int d \mathbf{Q} \int d \mathbf{P} \rho_{b}(\mathbf{Q}, \mathbf{P}) \exp \left[\frac{i}{\hbar} \int_{0}^{t} d \tau \delta U(\tau)\right],
$$

where $\mathbf{Q}=\left(Q_{1}, \ldots, Q_{N}\right)$ and $\mathbf{P}=\left(P_{1}, \ldots, P_{N}\right)$ are the classical nuclear coordinates and conjugated momenta, respectively, $\rho_{b}(\mathbf{Q}, \mathbf{P})$ is the phase space density that corresponds to the classical limit of $\hat{\rho}_{b}$, and $U(\tau)=U[\mathbf{Q}(\tau)]$, where $\mathbf{Q}(\tau)$ is obtained via a classical molecular dynamics simulation on the ground state potential energy surface with $\mathbf{Q}(\tau=0)=\mathbf{Q}$ and $\mathbf{P}(\tau=0)=\mathbf{P}$. Substituting the approximation in Eq. (15) into Eq. (10) then yields the following approximation for $\sigma_{g e}(t)$ :

$$
\frac{\sigma_{g e}(t)}{\sigma_{g e}(0)} \approx \exp \left\{i\left(\omega_{\mathrm{eg}}+\langle U\rangle_{b}^{\mathrm{cl}} / \hbar\right) t\right\} \times J_{b}^{\mathrm{cl}}(t)
$$

where

$$
\langle U\rangle_{b}^{\mathrm{cl}}=\int d \mathbf{Q} \int d \mathbf{P} \rho_{b}(\mathbf{Q}, \mathbf{P}) U(\mathbf{Q})
$$

is the classical limit of $\langle\hat{U}\rangle_{b}$.

\section{A NON-MARKOVIAN MASTER EQUATION FOR DEPHASING}

The analysis of homogeneity and Markovity can be most naturally carried out in terms of the system's equation of motion. In this section, we derive the equation of motion that governs the dynamics of $\widetilde{\sigma}_{g e}(t)$ as depicted by Eq. (14). To this end, we employ the following ansatz:

$$
\frac{d \widetilde{\sigma}_{g e}}{d t}=-\int_{0}^{t} d \tau \widetilde{K}_{b}(\tau) \widetilde{\sigma}_{g e}(t-\tau)
$$

The relationship between $\widetilde{K}_{b}(\tau)$ and $J_{b}(\tau)$ [Eq. (11)] can be established by taking the time derivative of Eq. (14), and writing it in terms of the ansatz in Eq. (18),

$$
\dot{J}_{b}(t)=-\int_{0}^{t} d \tau \widetilde{K}_{b}(\tau) J_{b}(t-\tau),
$$

where $\dot{J}_{b}(t)$ is the time derivative of $J_{b}(t)$. Taking the derivative with respect to $t$ of both sides of Eq. (19) and rearranging then leads to the following inhomogeneous Volterra equation of the second kind ${ }^{24}$ that relates $\widetilde{K}_{b}(\tau)$ to $J_{b}(\tau)$ :

$$
\tilde{K}_{b}(\tau)=-\ddot{J}_{b}(\tau)-\int_{0}^{\tau} d \tau^{\prime} \dot{J}_{b}\left(\tau-\tau^{\prime}\right) \tilde{K}_{b}\left(\tau^{\prime}\right),
$$

where $\ddot{J}_{b}(t)$ is the second time derivative of $J_{b}(t)$. Thus, given $J_{b}(\tau)$ as input, it is possible to solve Eq. (20) numerically for the memory kernel $\widetilde{K}_{b}(\tau){ }^{25}$

Equation (18) has the form of a generalized quantum master equation. Indeed, we have recently shown ${ }^{26}$ that this equation can also be derived by employing projection operator techniques within the framework of the NakajimaZwanzig formalism. ${ }^{27-30}$ This non-Markovian master equation can also be expressed in terms of the Schrödinger picture,

$$
\frac{d}{d t} \sigma_{g e}(t)=i\left(\omega_{\mathrm{eg}}+\langle\hat{U}\rangle_{b} / \hbar\right) \sigma_{g e}(t)-\int_{0}^{t} d \tau K_{b}(\tau) \sigma_{g e}(t-\tau),
$$

where the corresponding memory kernel function $K_{b}(\tau)$ $=\widetilde{K}_{b}(\tau) e^{i\left(\omega_{\mathrm{eg}}+\langle U\rangle_{b}\right) \tau}$ satisfies the following inhomogeneous Volterra equation of the second kind:

$$
\begin{aligned}
K_{b}(\tau)= & -e^{i\left(\omega_{\mathrm{eg}}+\langle\hat{U}\rangle_{b} / \hbar\right) \tau} \ddot{J}_{b}(\tau) \\
& -\int_{0}^{\tau} d \tau^{\prime} e^{i\left(\omega_{\mathrm{eg}}+\langle\hat{U}\rangle_{b} / \hbar\right)\left(\tau-\tau^{\prime}\right)} \dot{J}_{b}\left(\tau-\tau^{\prime}\right) K_{b}\left(\tau^{\prime}\right) .
\end{aligned}
$$

Equation (21) provides a convenient starting point for analyzing dynamical heterogeneity. To this end, it is important to note that Eq. (21) represents a family of nonMarkovian master equations, which differ with respect to the choice of the initial state of the nuclear DOF. More specifically, the memory kernel, $K_{b}(\tau)$, is explicitly dependent on the specific choice of $\hat{\rho}_{b}$. Further insight into the impact of dynamical heterogeneity can be obtained by considering the classical approximation for $J_{b}(t), J_{b}^{\mathrm{cl}}(t)$ [cf. Eq. (15)]. In the limit of homogeneous dynamics, $J_{b}^{b}(t)$ would be completely independent of $\rho_{b}^{\mathrm{cl}}(\mathbf{Q}, \mathbf{P})$ and can therefore be replaced by

$$
\begin{aligned}
J_{\mathrm{h}}^{\mathrm{cl}}(t)= & \int d \mathbf{Q} \int d \mathbf{P} \rho_{g}^{\mathrm{eg}}(\mathbf{Q}, \mathbf{P}) \\
& \times \exp \left\{\frac{i}{\hbar} \int_{0}^{t} d \tau[U(\mathbf{Q}[\tau])-U(\mathbf{Q})]\right\},
\end{aligned}
$$

where

$$
\begin{aligned}
\rho_{g}^{\mathrm{eq}}(\mathbf{Q}, \mathbf{P})= & \exp \left[-\beta H_{g}(\mathbf{Q}, \mathbf{P})\right] / \int d \mathbf{Q} \int d \mathbf{P} \\
& \times \exp \left[-\beta H_{g}(\mathbf{Q}, \mathbf{P})\right]
\end{aligned}
$$

is the equilibrium classical phase-space density that corresponds to the classical limit of $\hat{\rho}_{g}^{\text {eq }}$. The approximate homogeneous memory kernel can be obtained by substituting $J_{\mathrm{h}}^{\mathrm{cl}}(t)$ for $J_{b}(t)$ in Eq. (20) and solving it for the memory kernel. We denote the memory kernel that results from this procedure by $\tilde{K}_{\mathrm{h}}^{\text {cl }}(\tau)$. Upon substituting $\widetilde{K}_{\mathrm{h}}^{\text {cl }}(\tau)$ for $\widetilde{K}_{b}(\tau)$ in Eq. (18), we obtain the following homogeneous approximation of the non-Markovian master equation:

$$
\frac{d \widetilde{\sigma}_{g e}}{d t}=-\int_{0}^{t} d \tau \widetilde{K}_{\mathrm{h}}^{\mathrm{cl}}(\tau) \widetilde{\sigma}_{g e}(t-\tau) .
$$

The function $J_{\mathrm{h}}^{\mathrm{cl}}(t)$ should be clearly distinguished from the corresponding classical expression for the linear optical response function (ORF),

$$
\mathcal{J}^{\mathrm{l}}(t)=\int d \mathbf{Q} \int d \mathbf{P} \rho_{g}^{\mathrm{eq}}(\mathbf{Q}, \mathbf{P}) \exp \left\{i \int_{0}^{t} d \tau U(\mathbf{Q}[\tau]) / \hbar\right\} .
$$

$\mathcal{J}^{\mathrm{l}}(t)$ describes free-induction decay in the time domain, and its Fourier transform corresponds to the absorption spectrum. ${ }^{1}$ The difference between $J_{\mathrm{h}}^{\mathrm{cl}}(t)$ and $\mathcal{J}^{\mathrm{ll}}(t)$ lies in the integrands of the time integrals in Eqs. (23) and (25). In the case of $\mathcal{J}^{\mathrm{l}}(t)$ the integrand is given by $U(\mathbf{Q}[\tau])$, which spans 
a wide range of values at $\tau=0$ corresponding to the equilibrium distribution of solvent configurations. This so-called inhomogeneous broadening usually dominates the decay of $\mathcal{J}^{\mathrm{l}}(t)$, so that linear spectroscopy is insensitive to spectraldiffusion-induced dephasing. In contrast, the corresponding integrand in the case of $J_{\mathrm{h}}^{\mathrm{cl}}(t)$ is given by $U(\mathbf{Q}[\tau])-U(\mathbf{Q})$ and therefore vanishes at $\tau=0$. Thus, the decay of $J_{\mathrm{h}}^{\mathrm{cl}}(t)$ as a function of time is solely due to the dynamics of $U(\mathbf{Q}[\tau])$ (i.e., to spectral diffusion), which could be considerably slower in comparison to that of $\mathcal{J}^{\mathrm{l}}(t)$.

It is also interesting to compare the function $J_{\mathrm{h}}^{\mathrm{cl}}(t)$ with the classical frequency autocorrelation function,

$$
C_{U U}^{\mathrm{cl}}(t)=\int d \mathbf{Q} \int d \mathbf{P} \rho_{g}^{\mathrm{eq}}(\mathbf{Q}, \mathbf{P}) U(\mathbf{Q}) U(\mathbf{Q}[t]),
$$

which is often used for characterizing spectral diffusion processes. Both $J_{\mathrm{h}}^{\mathrm{cl}}(t)$ and $C_{U U}^{\mathrm{cl}}(t)$ are functions of only one time variable. Furthermore, like $J_{\mathrm{h}}^{\mathrm{cl}}(t), C_{U U}^{\mathrm{cl}}(t)$ involves an ensemble average over the equilibrium distribution of initial solvent configurations. However, using $C_{U U}^{\mathrm{cl}}(t)$ also assumes that the solvent obeys Gaussian statistics. Such an assumption is not made in the case of $J_{\mathrm{h}}^{\mathrm{cl}}(t)$.

The dynamics described by Eq. (24) is homogeneous but clearly non-Markovian. As such, it provides a convenient starting point for understanding the importance of nonMarkovian effects in solvation dynamics. In this context, the Markovian limit is defined as that in which the equation of motion becomes local in time, such that $d \widetilde{\sigma}_{g e}(t) / d t$ only depends on the value of $\widetilde{\sigma}_{g e}(t)$ at the same time. It should be noted that starting with the family of non-Markovian master equations represented by Eq. (21) would lead to a corresponding family of different Markovian equations. However, in this paper we only consider Markovity in the limit of homogeneous dynamics, which can be obtained by replacing $\widetilde{\sigma}_{g e}(t-\tau)$ by $\widetilde{\sigma}_{g e}(t)$ in Eq. (24). This yields the following Markovian master equation:

$$
\frac{d}{d t} \widetilde{\sigma}_{g e}(t)=-\widetilde{\gamma}_{\mathrm{h}}^{\mathrm{cl}}(t) \widetilde{\sigma}_{g e}(t)
$$

where

$$
\widetilde{\gamma}_{\mathrm{h}}^{\mathrm{cl}}(t)=\int_{0}^{t} d \tau \widetilde{K}_{\mathrm{h}}^{\mathrm{cl}}(\tau)
$$

It should be noted that Eq. (27) is expected to be accurate at short times, where $\widetilde{\sigma}_{g e}(t-\tau) \sim \widetilde{\sigma}_{g e}(t)$. Solving Eq. (27) for $\widetilde{\sigma}_{g e}(t)$ then yields

$$
\begin{aligned}
\frac{\widetilde{\sigma}_{g e}(t)}{\widetilde{\sigma}_{g e}(0)} & =\exp \left[-\int_{0}^{t} d t_{1} \widetilde{\gamma}_{\mathrm{h}}^{\mathrm{cl}}\left(t_{1}\right)\right] \\
& =\exp \left[-\int_{0}^{t} d t_{1} \int_{0}^{t_{1}} d t_{2} \widetilde{K}_{\mathrm{h}}^{\mathrm{cl}}\left(t_{2}\right)\right] .
\end{aligned}
$$

At times longer than the lifetime of $\widetilde{K}_{\mathrm{h}}^{\mathrm{cl}}(\tau), \widetilde{\gamma}_{\mathrm{h}}^{\mathrm{cl}}(t)$ becomes time independent, such that

$$
\frac{d}{d t} \widetilde{\sigma}_{g e}(t)=-\widetilde{\gamma}_{\mathrm{h}}^{\mathrm{cl}}(\infty) \widetilde{\sigma}_{g e}(t) \equiv-\left(\frac{1}{T_{2}}+i \Delta\right) \widetilde{\sigma}_{g e}(t),
$$

where

$$
\begin{aligned}
& \frac{1}{T_{2}} \equiv \operatorname{Re}\left[\widetilde{\gamma}_{\mathrm{h}}^{\mathrm{cl}}(\infty)\right]=\int_{0}^{\infty} d \tau \operatorname{Re}\left[\widetilde{K}_{\mathrm{h}}(\tau)\right], \\
& \Delta \equiv \operatorname{Im}\left[\widetilde{\gamma}_{\mathrm{h}}^{\mathrm{cl}}(\infty)\right]=\int_{0}^{\infty} d \tau \operatorname{Im}\left[\widetilde{K}_{\mathrm{h}}^{\mathrm{cl}}(\tau)\right] .
\end{aligned}
$$

Solving Eq. (30) for $\widetilde{\sigma}_{g e}(t)$ then yields

$$
\frac{\widetilde{\sigma}_{g e}(t)}{\widetilde{\sigma}_{g e}(0)}=\exp \left[-\left(\frac{1}{T_{2}}+i \Delta\right) t\right] \text {. }
$$

\section{CALCULATION OF PHOTON ECHO SIGNALS}

In this section we will outline the theoretical framework that will be used in Sec. V in order to calculate ORFs in liquid solutions. Here too, we will employ the classical approximation of Eq. (15).

We start out with the linear ORF given in Eq. (25). An equivalent expression for $\mathcal{J}^{\mathrm{l}}(t)$ is given by

$$
\mathcal{J}^{\mathrm{cl}}(t)=\int d U_{0} P\left(U_{0}\right) e^{i U_{0} t / \hbar} J_{b}^{\mathrm{cl}}\left(t ; U_{0}\right) .
$$

Here, $P\left(U_{0}\right)$ is the initial equilibrium probability density of $U(\mathbf{Q})$,

$$
P\left(U_{0}\right)=\int d \mathbf{Q} \int d \mathbf{P} \rho_{g}^{\mathrm{eq}}(\mathbf{Q}, \mathbf{P}) \delta\left[U(\mathbf{Q})-U_{0}\right],
$$

and $J_{b}^{\mathrm{cl}}\left(t ; U_{0}\right)$ is the same as in Eq. (15), except for the fact that its dependence on $U_{0}$ (the initial value of $U$ ) is now indicated explicitly.

In the case of truly homogeneous dynamics, one may substitute $J_{b}^{\mathrm{cl}}\left(t ; U_{0}\right)$ by $J_{\mathrm{h}}^{\mathrm{cl}}(t)$ [cf. Eq. (23)]. Since the latter is independent of $U_{0}$, one may then write $\mathcal{J}^{\mathrm{l}}(t)$ as a product of two factors,

$$
\mathcal{J}^{\mathrm{cl}}(t) \approx J_{\mathrm{inh}}^{\mathrm{cl}}(t) \times J_{\mathrm{h}}^{\mathrm{cl}}(t) .
$$

where

$$
J_{\mathrm{inh}}^{\mathrm{cl}}(t)=\int d U_{0} P\left(U_{0}\right) e^{i U_{0} t / \hbar} .
$$

The two Markovian limits of Eq. (35) can be obtained by replacing $J_{\mathrm{h}}^{\mathrm{cl}}(t)$ by $\widetilde{\sigma}_{g e}(t) / \widetilde{\sigma}_{g e}(0)$ from either Eq. (29) or (32).

It should be noted that the decay of $J_{\text {inh }}^{\text {cl }}(t)$ is due to the equilibrium distribution of $U(\mathbf{Q})$, whereas that of $J_{\mathrm{h}}^{\mathrm{cl}}(t)$ is due to the fluctuations in $U(\mathbf{Q})$ relative to its initial value. One may in fact define the homogeneous and inhomogeneous spectral line shapes in terms of the Fourier transforms of $J_{\mathrm{h}}^{\mathrm{cl}}(t)$ and $J_{\text {inh }}^{\mathrm{cl}}(t)$, respectively. While the inhomogeneous linewidth reflects the equilibrium distribution of solvent configurations, the inverse homogeneous linewidth provides a "characteristic" ensemble-averaged time scale of solvent fluctuations. For most disordered solvents such as liquids and 
glasses, one finds that the inhomogeneous line shape is typically much broader than the homogeneous line shape. Thus, $J_{\mathrm{h}}^{\mathrm{cl}}(t) \approx 1$ on the time scale where $J_{\text {inh }}^{\mathrm{cl}}(t)$ decays to zero, such that $\mathcal{J}^{\mathrm{l}}(t) \approx J_{\text {inh }}^{\mathrm{cl}}(t)$. The absorption line shape, which is given by the Fourier transform of $\mathcal{J}^{\mathrm{l}}(t)$, is therefore rather insensitive to spectral diffusion.

We next consider the classical ORF that corresponds to the three-pulse photon echo (3PE) experiment, $1,31,32$

$$
\begin{aligned}
\mathcal{R}\left(t_{1}, t_{2}, t\right)= & \int d \mathbf{Q} \int d \mathbf{P} \rho_{g}^{\mathrm{eq}}(\mathbf{Q}, \mathbf{P}) \\
& \times \exp \left\{i \int_{0}^{t_{1}} d \tau U(\tau) / \hbar-i \int_{t_{1}+t_{2}}^{t_{1}+t_{2}+t} d \tau U(\tau) / \hbar\right\} .
\end{aligned}
$$

Here, $t_{1}$ is the time interval between the first and second pulses, $t_{2}$ is the time interval between the second and third pulses, and the echo is detected a time period $t$ after the third pulse. 3PE signals are often homodyne detected. ${ }^{1}$ This means that one measures the echo intensity which is proportional to $\left|\mathcal{R}\left(t_{1}, t_{2}, t\right)\right|^{2}$. Furthermore, echo signals measured in liquid solutions and other disordered solvents are typically "smeared" over an extended time interval, rather than given by a sharp echo at $t=t_{1}$. The results of 3PE experiments are therefore often reported in terms of the integrated echo, which is given by

$$
I_{3 \mathrm{PE}}\left(t_{1}, t_{2}\right)=\int_{0}^{\infty} d t\left|\mathcal{R}\left(t_{1}, t_{2}, t\right)\right|^{2} .
$$

We also note that the two-pulse photon echo (2PE) is a special case of the $3 \mathrm{PE}$, where $t_{1}$ is the time interval between the first and second pulses and $t_{2}=0$ in Eq. (37).

The 3PE ORF can also be written in the following form:

$$
\begin{aligned}
\mathcal{R}\left(t_{1}, t_{2}, t\right)= & \int d U_{0} \int d U_{2} P\left(U_{0}, U_{2} ; t_{1}+t_{2}\right) \\
& \times e^{i U_{0} t_{1} / h-i U_{2} t / \hbar} J_{b}^{\mathrm{cl}}\left(t_{1} ; U_{0}\right)\left[J_{b}^{\mathrm{cl}}\left(t ; U_{2}\right)\right]^{*}
\end{aligned}
$$

Here, $P\left(U_{0}, U_{2} ; t_{1}+t_{2}\right)$ is the probability density of finding $U=U_{0}$ and $U=U_{2}$ a time interval $t_{1}+t_{2}$ apart. It should be noted that in this experiment, the coherence is stored as population for a period of time $t_{2}$ between the second and third pulses. Thus, spectral diffusion during this time does not lead to dephasing, although it does affect the initial distribution of solvent configuration for the dephasing process that follows the third pulse.

In the case of truly homogeneous dynamics, one may substitute $J_{b}^{\mathrm{cl}}\left(t_{1} ; U_{0}\right)$ and $J_{b}^{\mathrm{cl}}\left(t ; U_{2}\right)$ by $J_{\mathrm{h}}^{\mathrm{cl}}\left(t_{1}\right)$ and $J_{\mathrm{h}}^{\mathrm{cl}}(t)$, respectively. As a result, one may write $\mathcal{R}\left(t_{1}, t_{2}, t\right)$ as a product of two factors,

$$
\mathcal{R}\left(t_{1}, t_{2}, t\right)=R_{\mathrm{inh}}^{\mathrm{cl}}\left(t_{1}, t_{2}, t\right) \times J_{\mathrm{h}}^{\mathrm{cl}}\left(t_{1}\right)\left[J_{\mathrm{h}}^{\mathrm{cl}}(t)\right]^{*},
$$

where

$$
\begin{aligned}
R_{\mathrm{inh}}^{\mathrm{cl}}\left(t_{1}, t_{2}, t\right)= & \int d U_{0} \int d U_{2} P\left(U_{0}, U_{2} ; t_{1}+t_{2}\right) \\
& \times e^{i U_{0} t_{1} / \hbar-i U_{2} t / \hbar} .
\end{aligned}
$$

The two Markovian limits of Eq. (40) can be obtained by replacing $J_{\mathrm{h}}^{\mathrm{cl}}\left(t_{1}\right)$ and $J_{\mathrm{h}}^{\mathrm{cl}}(t)$ by the corresponding expressions from either Eq. (29) or (32).

The two factors on the right-hand side of Eq. (40) represent two opposing effects. The first factor, $R_{\text {inh }}^{\mathrm{cl}}\left(t_{1}, t_{2}, t\right)$, leads to an echo centered at $t=t_{1}$, which diminishes as $t_{2}$ is increased due to spectral diffusion (but not dephasing!) during the time interval between the second and third pulses $\left(t_{2}\right)$. The second term represents dephasing processes that take place during the time intervals between the first and second pulses $\left(t_{1}\right)$ and between the third pulse and echo detection $(t)$, and which diminish the echo signal.

\section{APPLICATION TO NONPOLAR LIQUID SOLUTION}

In this section, we apply the theoretical framework developed in the previous sections for studying Markovity and homogeneity of dephasing in the case of a two-state chromophore solvated in a monoatomic liquid. The potential energy surfaces of the ground and excited electronic states are assumed to be pairwise additive, such that

$$
V_{\alpha}=\sum_{k} v_{\alpha}\left(r_{k}\right)+\sum_{k<j} v_{s}\left(r_{k j}\right)
$$

Here, $\alpha=g, e$, and $r_{k}$ is the distance between the chromophore and the $k$ th solvent atom and $r_{k j}$ is the distance between the $k$ th and $j$ th solvent atoms. It should be noted that the chromophore-solvent interactions in the excited state are assumed to be different from those in the ground state, whereas the solvent-solvent interactions in both states are assumed to be the same.

The actual pair potentials $v_{g}(r), v_{e}(r)$, and $v_{s}(r)$ were adopted from Refs. 21 and 32, where they were assumed to take the form of Lennard-Jones (LJ) potentials,

$$
v_{j}(r)=4 \epsilon_{j}\left[\left(\frac{\sigma_{j}}{r}\right)^{12}-\left(\frac{\sigma_{j}}{r}\right)^{6}\right] .
$$

We also assume that $v_{g}(r)=v_{s}(r)$, with LJ parameters that correspond to liquid argon $\left(\epsilon / k_{B}=119.8 \mathrm{~K}\right.$ and $\sigma$ $=0.3405 \mathrm{~nm}) \cdot v_{e}(r)$ differs from $v_{g}(r)$ in the value of the LJ parameter $\sigma$, such that $\sigma_{e}=(1+\lambda) \sigma_{g}$, with $\lambda=0.06 .^{21,32} \mathrm{We}$ also assume that the chromophore mass is the same as that of the solvent atoms and equal to the mass of an argon atom.

Molecular dynamics (MD) simulations were carried out at temperature $T=128.2 \mathrm{~K}$ and density $\rho=17.98 \mathrm{~nm}^{-3}$ (the corresponding reduced LJ temperature and density are given by $T^{*}=k_{B} T / \epsilon_{s}=1.07$ and $\rho^{*}=\rho \sigma_{s}^{3}=0.71$, respectively). Simulations were preformed with 108 atoms in a cubical simulation box with standard periodic boundary conditions (minimum image convention). Time propagation was carried out by the velocity Verlet method. ${ }^{33}$ The system was equilibrated over $10^{6}$ time steps, each of length of $4 \mathrm{fs}$, by the velocity rescaling method. ${ }^{33}$ Converged results for $J_{\mathrm{h}}^{\mathrm{cl}}(t)$ and $\mathcal{J}^{\mathrm{cl}}(t)$ were obtained by averaging over 100 trajectories, each of length of $10 \mathrm{ps}$. Converged results for $J_{\mathrm{inh}}^{\mathrm{cl}}(t)$ required some- 


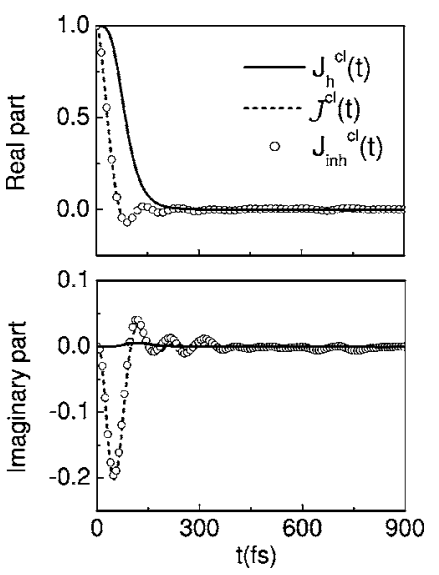

FIG. 1. Real part (upper panel) and imaginary part (lower panel) of $J_{\mathrm{h}}^{\mathrm{cl}}(t)$ (solid line), $\mathcal{J}^{\mathrm{l}}(t)$ (dashed line), and $J_{\text {inh }}^{\mathrm{cl}}(t)$ (circles).

what less averaging. In the following figures, the error bars are comparable to the thickness of the lines and therefore not shown explicitly.

The real and imaginary parts of the function $J_{\mathrm{h}}^{\mathrm{cl}}(t)[\mathrm{Eq}$. (23)] as obtained from the MD simulations is shown in Fig. 1. Also shown are the real and imaginary parts of $J_{\text {inh }}^{\mathrm{cl}}(t)[\mathrm{Eq}$. (36)] and $\mathcal{J}^{\mathrm{cl}}(t)$ [Eq. (25)]. As expected, $\mathcal{J}^{\mathrm{l}}(t)$ almost coincides with $J_{\text {inh }}^{\mathrm{cl}}(t)$ and they both decay more rapidly than $J_{\mathrm{h}}^{\mathrm{cl}}(t)$. This is consistent with the fact that the absorption line shape is inhomogeneously broadened. Indeed, Fig. 2 shows that the absorption line shape, given by the Fourier transform of $\mathcal{J}^{\mathrm{l}}(t)$, essentially coincides with the inhomogeneous line shape, given by the Fourier transform of $J_{\text {inh }}^{\mathrm{cl}}(t)$, and that both are significantly broader than the homogeneous line shape, which is defined by the Fourier transform of $J_{\mathrm{h}}^{\mathrm{cl}}(t)$. It is also interesting to note that the shapes of the homogeneous and inhomogeneous lines are qualitatively different, with the former clearly more symmetrical than the latter.

The real and imaginary parts of the memory kernel $\widetilde{K}_{\mathrm{h}}^{\mathrm{cl}}(t)$, as obtained by numerically solving Eq. (20) with $J_{\mathrm{h}}^{\mathrm{cl}}(t)$ from Fig. 1 as input, are shown in Fig. 3. The real part is responsible for dephasing, while the imaginary part leads to a phase shift (which turns out to be rather small for the model considered here). Figure 4 shows that $\widetilde{\sigma}_{g e}(t)$ obtained by substituting this memory kernel into Eq. (24), and numerically solving it for $\widetilde{\sigma}_{g e}(t)$, coincides with the exact solution [Eq. (16)]. The Markovian approximation in Eq. (27) is

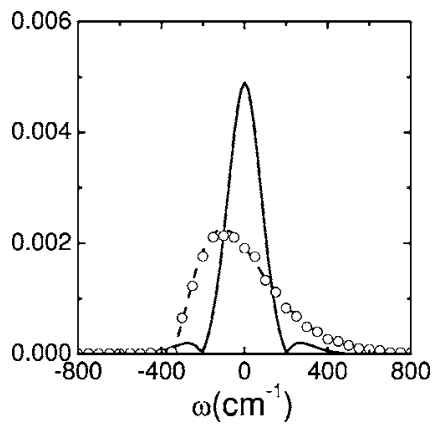

FIG. 2. The homogeneous line shape [Fourier transforms of $J_{\mathrm{h}}^{\mathrm{cl}}(t)$, solid line], absorption line shape [Fourier transforms of $\mathcal{J}^{\mathrm{l}}(t)$, dashed line], and inhomogeneous line shape [Fourier transform of $J_{\text {inh }}^{\mathrm{cl}}(t)$, circles].

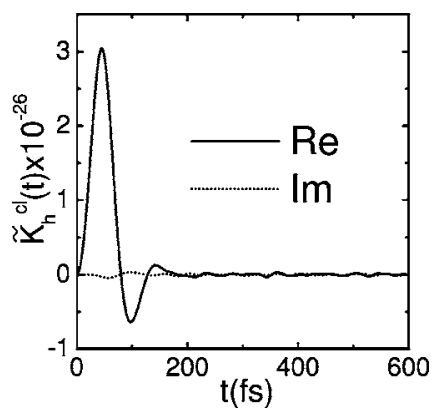

FIG. 3. The real part (solid line) and imaginary part (dotted line) of the homogeneous memory kernel $K_{\mathrm{h}}^{\mathrm{cl}}(t)$.

seen to coincide with the exact solution at short times, but decays too slowly at longer times. The decay of $\widetilde{\sigma}_{g e}(t)$ predicted by the Markovian approximation in Eq. (30) is qualitatively different than the exact result at all times, although the actual time scale of the overall decay is reasonably accurate.

In Fig. 5, we show the homodyne-detected integrated 2PE signal as a function of $t_{1}$, the time interval between the first and second pulses. The exact homodyne-detected integrated 2PE was calculated via Eq. (37) and is given by the solid line in Fig. 5. Assuming homogeneous dynamics [Eq. (40)] yields the prediction given by the dashed line which is somewhat lower than the exact result. However, assuming homogeneous dynamics does not alter the qualitative behavior of the $2 \mathrm{PE}$ signal as a function of $t_{1}$ (although the peak location is shifted to a somewhat shorter time). Adding the Markovian approximation in Eq. (27) leads to a result which is very similar to that obtained from the non-Markovian treatment. This is consistent with the fact that the $2 \mathrm{PE}$ signal is dominated by the short time dynamics of $\widetilde{\sigma}_{g e}(t)$, for which Eq. (27) provides an excellent approximation (cf. Fig. 4). At the same time, the Markovian approximation in Eq. (30) is seen to provide a qualitatively incorrect $2 \mathrm{PE}$ signal.

Finally, in Fig. 6 we show the homodyne-detected integrated $3 \mathrm{PE}$ as a function of $t_{1}$ and $t_{2}$. We once again find that assuming homogeneous dynamics lowers the signal and

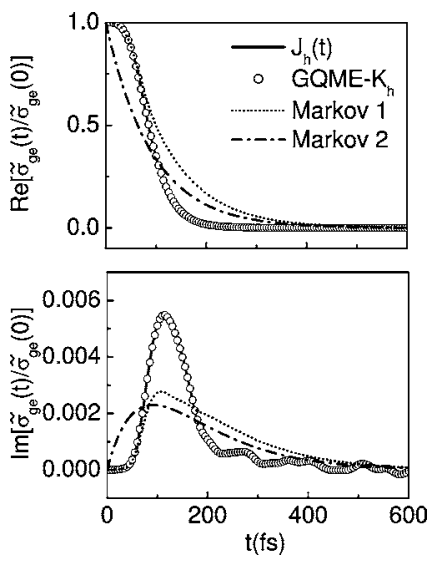

FIG. 4. Real part (upper panel) and imaginary part (lower panel) of $\bar{\sigma}_{g e}(t) / \bar{\sigma}_{g e}(0)$ as obtained from $J_{\mathrm{h}}(t)$ (solid line), the non-Markovian master [Eq. (18)] equation with a homogeneous memory kernel (circles), and its Markovian approximations in Eqs. (29) (dotted line) and (32) (dotteddashed line). 


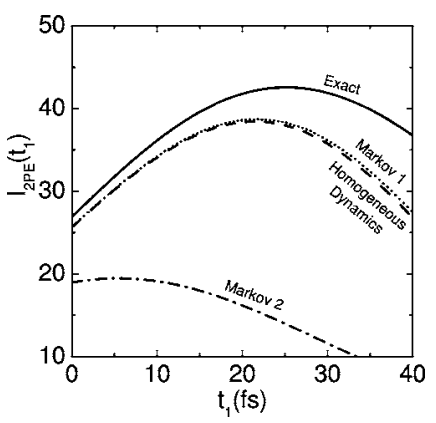

FIG. 5. The homodyne detected integrated 2PE signal as a function of $t_{1}$ as obtained via Eq. (37) (solid line), Eq. (40) (dashed line), and the Markovian approximations in Eqs. (29) (dotted line) and (32) (dotted-dashed line).

shifts the peak to slightly shorter times, but does not otherwise change its qualitative behavior. Here too, the Markovian approximation in Eq. (27) predicts a signal which is very similar to that predicted by the non-Markovian treatment, while the Markovian approximation in Eq. (30) is seen to provide a qualitatively incorrect echo signal.

\section{SUMMARY}

In this paper, we have developed a theoretical framework for analyzing the extent to which the dynamics of electronic dephasing in liquid solutions is homogeneous and Markovian. It should be noted that the proposed scheme is very general and can be applied to practically any disordered system of interest, including glasses, polymer melts, and biosystems. The proposed scheme relies on a description of the dephasing dynamics in terms of a non-Markovian master equation [Eq. (18)], where the system and bath correspond to the electronic and nuclear DOFs, respectively. The central quantity in this equation is the memory kernel function, $K_{b}(\tau)$. Dynamical heterogeneity is manifested by the fact that $K_{b}(\tau)$ is explicitly dependent on the initial solvent configuration. Furthermore, $K_{b}(\tau)$ can be obtained by numerically solving the inhomogeneous Volterra equation of the second kind in Eq. (20), which requires as input the quantity $J_{b}(\tau)$. The latter can be estimated by $J_{b}^{\mathrm{cl}}(\tau)$ which can be obtained from a classical MD simulation [cf. Eq. (15)]. In the limit of homogeneous dynamics, $J_{b}^{\mathrm{cl}}(\tau)$ becomes independent of the initial solvent configuration and can therefore be replaced by $J_{\mathrm{h}}^{\mathrm{cl}}(\tau)$ [cf. Eq. (23)]. The quantity $J_{\mathrm{h}}^{\mathrm{cl}}(\tau)$ also provides a convenient new and rather general way for defining homogeneous broadening. Two types of Markovian limits of the above-mentioned non-Markovian master equation can also be conveniently defined, with either time-dependent or timeindependent dephasing coefficients [cf. Eqs. (27) and (30), respectively].

The application of the above results to the case of a monoatomic liquid solvent reveals that the dynamics is rather heterogeneous on the time scale relevant for most ultrafast experiments. Nevertheless, this dynamical heterogeneity does not seem to alter the main quantitative features of photon echo signals, which are often employed for characterizing the time scale of electronic dephasing. It should be noted, however, that the same conclusion may not hold in the case of more disordered systems such as glasses and biosys-
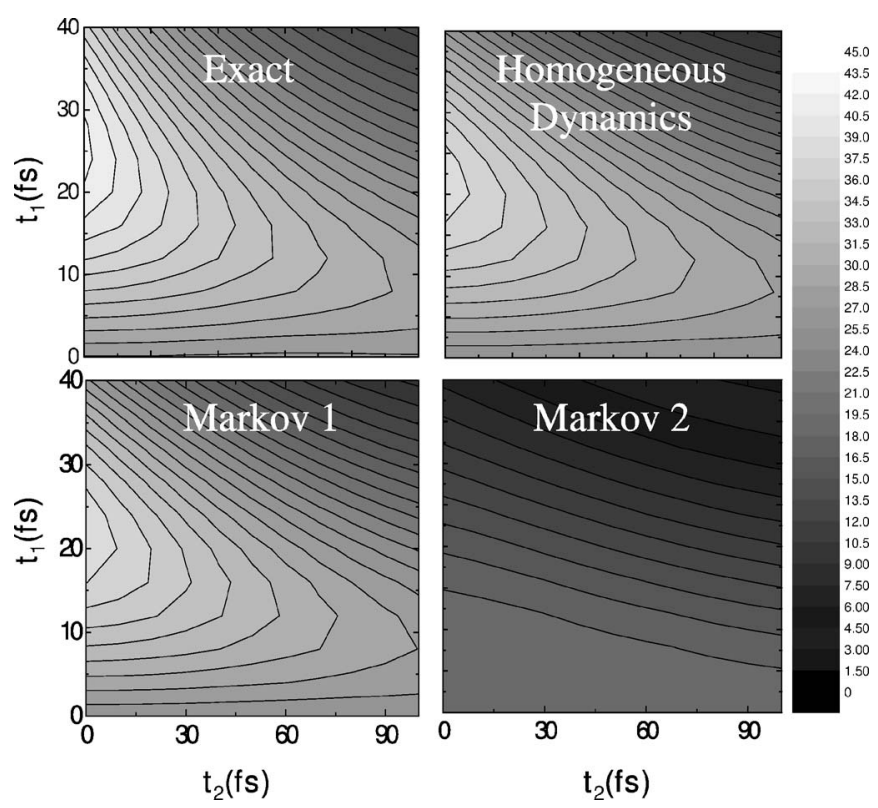

FIG. 6. The homodyne detected integrated 3PE signal as a function of $t_{1}$ and $t_{2}$ as obtained via Eq. (37) (upper left), Eq. (40) (upper right), and the Markovian approximations in Eqs. (29) (lower left) and (32) (lower right).

tems, where dynamical heterogeneity may have a more pronounced signature. The non-Markovian nature of the dephasing dynamics is also observed to have almost no effect on the photon echo signal. The latter can be reproduced rather well by using the Markovian master equation with a timedependent dephasing coefficient [Eq. (27)]. This can be traced back to the sensitivity of the echo to the dephasing dynamics at very short times, where it is described rather well by Eq. (27). At the same time, using the Markovian master equation with a time-independent dephasing coefficient [Eq. (30)] was seen to lead to qualitatively different results.

The methodology outlined here can be extended in a variety of ways. First, one may replace the classical approximation used for calculating $J_{\mathrm{h}}^{\mathrm{cl}}(t)$ by more rigorous semiclassical and mixed quantum-classical treatments. ${ }^{34-38}$ Second, one may further explore the extent of dynamical heterogeneity by examining the underlying distributions of $J_{b}(t)$ and $K_{b}(t)$. Third, one may attempt to calculate the memory kernel for a driven chromophore (i.e., with an explicitly timedependent Hamiltonian). Finally, one may employ the procedures outlined above in order to investigate dynamical heterogeneity and non-Markovity in more disordered solvents, such as glasses and biomolecules. Work on these issues is underway in our group and will be reported in future publications.

\section{ACKNOWLEDGMENTS}

This project was supported by the National Science Foundation through Grant No. CHE-306695 and the Petroleum Research Fund.

\footnotetext{
${ }^{1}$ S. Mukamel, Principles of Nonlinear Optical Spectroscopy (Oxford, New York, 1995).

${ }^{2}$ G. R. Fleming and M. Cho, Annu. Rev. Phys. Chem. 47, 109 (1996).

${ }^{3}$ W. P. de Boeij, M. S. Pshenichnikov, and D. A. Wiersma, Annu. Rev.
} 
Phys. Chem. 49, 99 (1998).

${ }^{4}$ A. B. Myers, Annu. Rev. Phys. Chem. 49, 267 (1998).

${ }^{5}$ R. M. Stratt and M. Maroncelli, J. Phys. Chem. 100, 12981 (1996).

${ }^{6}$ P. Vöhringer, D. C. Arnett, T.-S. Yang, and N. F. Scherer, Chem. Phys. Lett. 237, 387 (1995).

${ }^{7}$ D. Hsu and J. L. Skinner, J. Chem. Phys. 81, 5471 (1984).

${ }^{8}$ P. D. Reilly and J. L. Skinner, Phys. Rev. Lett. 71, 4257 (1993).

${ }^{9}$ P. D. Reilly and J. L. Skinner, J. Chem. Phys. 102, 1540 (1995).

${ }^{10}$ E. Geva, P. D. Reilly, and J. L. Skinner, Acc. Chem. Res. 29, 579 (1996).

${ }^{11}$ E. Geva and J. L. Skinner, Mol. Cryst. Liq. Cryst. Sci. Technol., Sect. A 291, 73 (1996).

${ }^{12}$ E. Geva and J. L. Skinner, Chem. Phys. Lett. 287, 125 (1998).

${ }^{13}$ M. Orrit, J. Bernard, and R. I. Personov, J. Phys. Chem. 97, 10256 (1993).

${ }^{14}$ W. E. Moerner, Acc. Chem. Res. 29, 563 (1996).

${ }^{15}$ T. M. Chang and J. L. Skinner, Physica A 193, 483 (1993).

${ }^{16}$ E. T. J. Nibbering, D. A. Wiersma, and K. Duppen, Phys. Rev. Lett. 66, 2464 (1991).

${ }^{17}$ W. P. de Boeij, M. S. Pshenichnikov, K. Duppen, and D. A. Wiersma, Chem. Phys. Lett. 224, 243 (1994).

${ }^{18}$ W. P. de Boeij, M. S. Pshenichnikov, and D. A. Wiersma, J. Chem. Phys. 105, 2953 (1996).

${ }^{19}$ P. Cong, Y. J. Yan, H. P. Deuel, and J. D. Simon, J. Chem. Phys. 100, 7855 (1994).

${ }^{20}$ J. G. Saven and J. L. Skinner, J. Chem. Phys. 99, 4391 (1993).
${ }^{21}$ M. D. Stephens, J. G. Saven, and J. L. Skinner, J. Chem. Phys. 106, 2129 (1997).

${ }^{22}$ S. A. Egorov, E. Rabani, and B. J. Berne, J. Chem. Phys. 108, 1407 (1998).

${ }^{23}$ S. A. Egorov, E. Rabani, and B. J. Berne, J. Chem. Phys. 110, 5238 (1999).

${ }^{24}$ P. M. Morse and H. Feshbach, Methods of Theoretical Physics (McGrawHill, New York, 1953), Pt. 1.

${ }^{25}$ W. H. Press, B. P. Flannery, S. A. Teukolsky, and W. T. Vetterling, $\mathrm{Nu}-$ merical Recipes (Cambridge University Press, Cambridge, 1986).

${ }^{26}$ M.-L. Zhang, B. J. Ka, and E. Geva, J. Chem. Phys. 125, 044106 (2006).

${ }^{27}$ S. Nakajima, Prog. Theor. Phys. 20, 948 (1958).

${ }^{28}$ R. Zwanzig, Lect. Theor. Phys. 3, 106 (1960).

${ }^{29}$ R. Zwanzig, J. Chem. Phys. 33, 1338 (1960).

${ }^{30}$ R. Zwanzig, Physica (Amsterdam) 30, 1109 (1964).

${ }^{31}$ K. F. Everitt, E. Geva, and J. L. Skinner, J. Chem. Phys. 114, 1326 (2001).

${ }^{32}$ K. F. Everitt and J. L. Skinner, Chem. Phys. 266, 197 (2001).

${ }^{33}$ M. P. Allen and D. J. Tildesley, Computer Simulation of Liquids (Clarendon, Oxford, 1987).

${ }^{34}$ N. Makri, Annu. Rev. Phys. Chem. 50, 167 (1999).

${ }^{35}$ Q. Shi and E. Geva, J. Chem. Phys. 120, 10647 (2004).

${ }^{36}$ Q. Shi and E. Geva, J. Phys. Chem. A 108, 6109 (2004).

${ }^{37}$ Q. Shi and E. Geva, J. Chem. Phys. 121, 3393 (2004).

${ }^{38}$ Q. Shi and E. Geva, J. Chem. Phys. 122, 064506 (2005). 